\title{
Numerical investigation of nonlinear generalized regularized long wave equation via delta-shaped basis functions
}

\author{
Ömer Oruç* \\ Ĕ̆il Vocational and Technical Anatolian High School, Diyarbakır, Turkey \\ omeroruc0@gmail.com
}

\section{ARTICLE INFO}

Article History:

Received 04 November 2019

Accepted 25 May 2020

Available 01 July 2020

Keywords:

Delta-Shaped basis functions

Nonlinear PDE

GRLW equation

Meshless method

Numerical solution

AMS Classification 2010:

65M70; 65 N35

\section{ABSTRACT}

In this study we will investigate generalized regularized long wave (GRLW) equation numerically. The GRLW equation is a highly nonlinear partial differential equation. We use finite difference approach for time derivatives and linearize the nonlinear equation. Then for space discretization we use deltashaped basis functions which are relatively few studied basis functions. By doing so we obtain a linear system of equations whose solution is used for constructing numerical solution of the GRLW equation. To see efficiency of the proposed method four classic test problems namely the motion of a single solitary wave, interaction of two solitary waves, interaction of three solitary waves and Maxwellian initial condition are solved. Further, invariants are calculated. The results of numerical simulations are compared with exact solutions if available and with finite difference, finite element and some collocation methods. The comparison indicates that the proposed method is favorable and gives accurate results.

\section{Introduction}

Consider following generalized equation

$$
\begin{aligned}
u_{t}+\alpha u_{x}+\epsilon\left(u^{p}\right)_{x}-\mu u_{x x t} & -\gamma u_{x x}=0, \\
& -\infty<x<\infty, t>0
\end{aligned}
$$

in which $t$ is time, $x$ is spatial variable and $u$ is the amplitude, and $\alpha \geq 0, \epsilon \geq 0, \mu \geq 0, \gamma \geq 0$, $p \geq 2$. The Eq. (11) presents a lot of mathematical models according to the values of $\alpha, \epsilon, \mu, \gamma[1$ for instance :

- if $\alpha=0, \epsilon=0, \mu=0, \gamma \neq 0$ then Eq. (1) corresponds to heat equation,

- if $\alpha \neq 0, \epsilon=0, \mu=0, \gamma=0$ then Eq. (1) corresponds to wave equation,

- if $\alpha=0, \epsilon \neq 0, \mu=0, \gamma \neq 0, p=2$ then Eq. (1) corresponds to viscous Burgers' equation,
- if $\alpha=1, \epsilon \neq 0, \mu \neq 0, \gamma=0, p=2$ then Eq. (1) corresponds regularized long wave (RLW) equation,

- if $\alpha=1, \epsilon \neq 0, \mu \neq 0, \gamma=0, p>2$ then Eq. (1) corresponds generalized regularized long wave (GRLW) equation.

In this paper, we will study GRLW equation numerically. The GRLW equation was first proposed by Peregrine 2, 3, for description of an undular bore and then by Benjamin et al. 4] GRLW equation suggested as a model for long waves with small amplitudes on the surface of water in a channel. Since the GRLW equation can be a model for a lot of real life phenomena such as plasma waves 5 and shallow water waves 2 it is crucial to develop efficient methods for solving this equation. Since analytical solutions of the GRLW equation are available only for limited initial and boundary conditions it is inevitable for 
looking at numerical methods. Due to highly nonlinear structure of the GRLW equation, developing efficient numerical methods for this equation also is a challenging work.

GRLW equation includes RLW and modified RLW equation for certain values of $p$. There are a vast of studies related to both RLW and modified RLW equations, see for example [6-14] and references therein. On the other hand for the GRLW equation literature is not so rich. But, nevertheless there are some studies related to the GRLW equation. For example, the GRLW equation has been solved using Sinc-collocation method [16], Wang et al. [17] used a meshless method for the GRLW equation, element-free kpRitz method has been used by Guo et al. [18] for solving the GRLW equation, Kang et al. [19] used a second-order Fourier pseudospectral method for the GRLW equation, compact finite difference method and finite difference method have been used in [20, 21], respectively. Roshan [22, used a Petrov-Galerkin method for the GRLW equation. B-spline finite element method has been used in [23], a collocation method with cubic Bsplines is used in [6], Karakoc and Zeybek [24] used septic B-spline collocation method, more recently local momentum-preserving algorithms [25] are developed for the GRLW equation.

We will investigate numerical solution of the GRLW equation given in following form;

$$
u_{t}+u_{x}+p(p+1) u^{p} u_{x}-\mu u_{x x t}=0, \quad a \leq x \leq b
$$

with Dirichlet boundary conditions $u(a, t)=$ $u(b, t)=0$ by employing finite difference and delta-shaped basis functions.

The paper is organized as follows. In Section 2, a brief information about delta-shaped basis functions is given. In section 3 , time discretization with finite difference and space discretization with delta-shaped basis functions are described. The results of numerical simulations are presented in Section 4. Finally, the paper is concluded in Section 5 .

\section{Delta-shaped basis functions}

Delta-shaped basis functions (DBFs) have been derived by Reutskiy [26] from Fourier series of Dirac-delta function and were used for simulating a set of scattered data in both regular and irregular domains successfully. Since then DBFs have been used in some studies for numerical solution of partial differential equations. For instance, DBFs are used for solving Helmholtz-type equations in [27, 28], Hon and Yang used DBFs for default barrier model [29], one-dimensional Stefan problems are solved by DBFs [30, numerical solution of the Schrödinger equations are obtained by using DBFs [31], DBFs are used for solving ill-posed nonhomogeneous elliptic boundary value problems 32, recently a pseudo spectral method based on DBFs is developed in 33. for solving modified Burgers equation. We briefly introduce delta-shaped basis functions, in the sequel [29,31]. Consider following Sturm-Liouville eigenvalue problem

$$
\left\{\begin{array}{l}
-\frac{d^{2} \phi}{d x^{2}}=\lambda \phi, \\
\phi(-1)=\phi(1)=0 .
\end{array} \quad x \in(-1,1)\right.
$$

Let $\left(\phi_{n}(x), \lambda_{n}\right)$ be a solution to the above SturmLiouville eigenvalue problem. Clearly, $\phi_{n}(x)=$ $\sin \left(n \pi \frac{x+1}{2}\right), \lambda_{n}=\left(\frac{n \pi}{2}\right)^{2}$ and further

$$
\int_{-1}^{1} \phi_{m}(x) \phi_{n}(x) d x=\delta_{m n}= \begin{cases}1, & m=n, \\ 0, & m \neq n .\end{cases}
$$

That means, eigenfunctions $\left\{\phi_{n}(x)\right\}_{n=1}^{\infty}$ form an orthogonal system on interval $[-1,1]$ and furthermore Dirac's delta function can be expressed as follows

$$
\delta(x-\xi)=\sum_{n=1}^{\infty} \phi_{n}(\xi) \phi_{n}(x) .
$$

The series in Eq. (3), can be used with some regularization techniques [30] to derive smooth deltashaped function $I_{M, \chi}(x, \xi)$. Otherwise the series in Eq. (3) diverges at any point in the interval $[-1,1]$ [26]. Here we consider Riesz regularization approach and thus the regularized delta-shaped functions are in the following form

$$
I_{M, \chi}(x, \xi)=\sum_{n=1}^{M}\left(1-\frac{n^{2}}{(M+1)^{2}}\right)^{\chi} \phi_{n}(\xi) \phi_{n}(x) \text {. }
$$

The parameters $M$ and $\chi$ may be think of as shape parameters since they form the properties of delta-shaped functions. The parameter $M$ is responsible for scaling, as $M$ increases the support of basis function decreases. This can be seen in left column of Fig. 1. The parameter $\chi$ responsible for regularizing, if $\chi=0$ i.e. when there is no regularization, basis function shows oscillating behavior on its support. On the other hand if $\chi$ increases basis function gets smoother. We show this situation in right column of Fig. 1. We should note that choosing optimal values of shape 

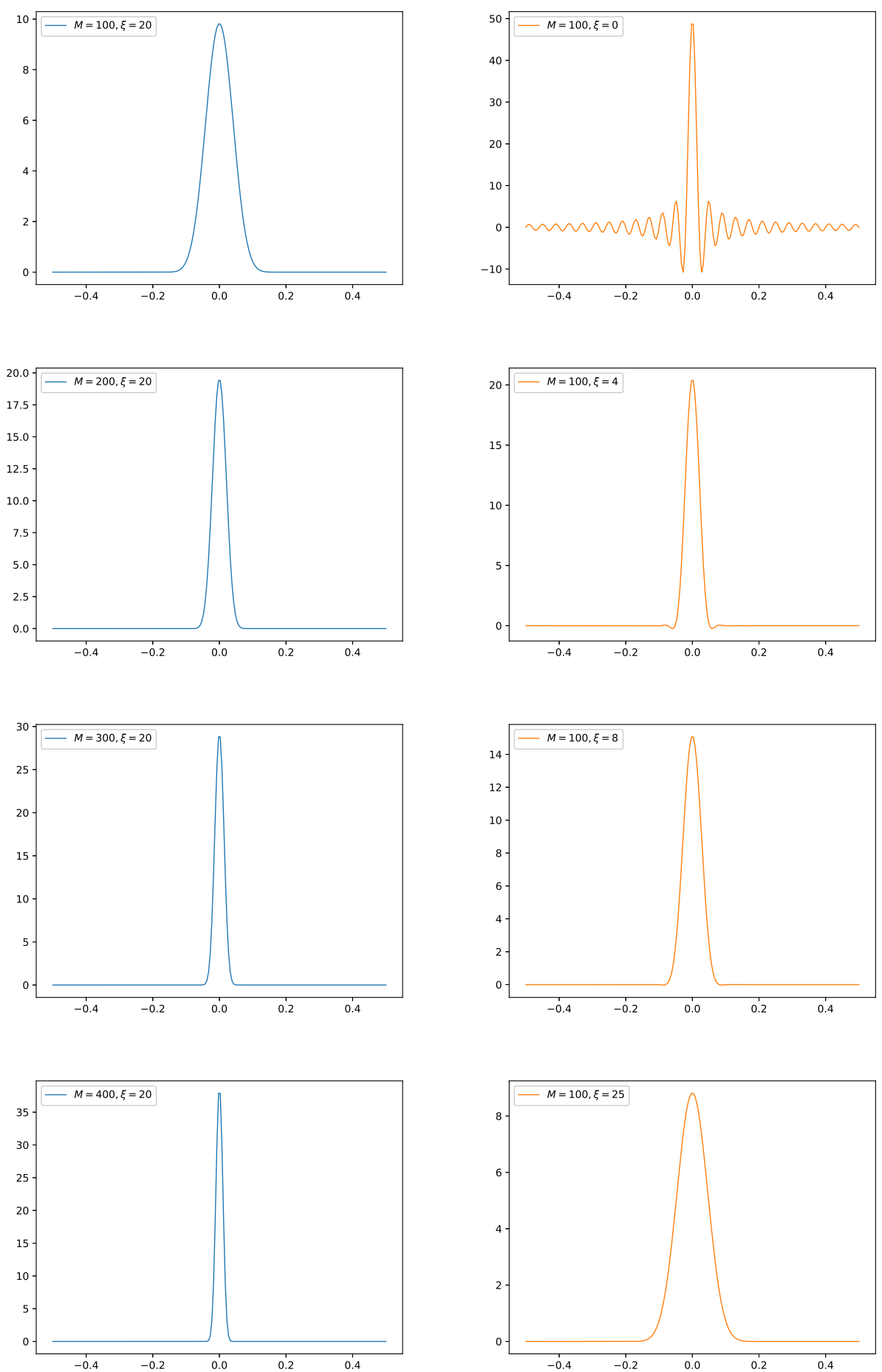

Figure 1. Effect of $M$ and $\chi$ with center $\xi=0$ on delta-shaped functions.

parameters for delta-shaped functions is still an open problem.

\section{Solution method for GRLW equation}

In this section, we describe time discretization and space discretization for the GRLW equation. We start with time discretization.

\subsection{Time discretization by finite differences}

We take GRLW equation as

$$
u_{t}+u_{x}+\epsilon u^{p} u_{x}-\mu u_{x x t}=0, \quad a \leq x \leq b, t>0
$$

with initial condition 


$$
u(x, 0)=f(x),
$$

and boundary conditions

$$
u(a, t)=g_{1}(t), u(b, t)=g_{2}(t), t \geq 0,
$$

where $\epsilon=p(p+1)>0, \mu>0$ and $f(x), g_{1}(t)$ and $g_{2}(t)$ are prescribed functions. We use forward Euler formula for time derivatives and utilize $\theta$-weighted $(0 \leq \theta \leq 1)$ scheme between time levels $j$ and $j+1$ as

$$
\begin{aligned}
\frac{u^{j+1}-u^{j}}{\Delta t}+ & \theta\left[\left(u_{x}\right)^{j+1}+\epsilon\left(u^{p} u_{x}\right)^{j+1}\right] \\
+ & (1-\theta)\left[\left(u_{x}\right)^{j}+\epsilon\left(u^{p} u_{x}\right)^{j}\right] \\
& -\frac{\mu}{\Delta t}\left(\left(u_{x x}\right)^{j+1}-\left(u_{x x}\right)^{j}\right)=0
\end{aligned}
$$

where $\Delta t$ is time step size and $t^{j+1}=t^{j}+\Delta t$, $u^{j+1}=u\left(x, t^{j+1}\right)$. Following [16], the nonlinear term $\left(u^{p} u_{x}\right)^{j+1}$ can be linearized as

$$
\begin{aligned}
\left(u^{p} u_{x}\right)^{j+1} & \simeq\left(u^{p} u_{x}\right)^{j}+\Delta t\left[\left(u_{t}^{p}\right)^{j} u_{x}^{j}+\left(u^{p}\right)^{j}\left(u_{x t}\right)^{j}\right] \\
& +\mathcal{O}\left(\Delta t^{2}\right) \\
& =\left(u^{p} u_{x}\right)^{j} \\
& +\Delta t\left[\frac{\left(u^{p}\right)^{j+1}-\left(u^{p}\right)^{j}}{\Delta t} u_{x}^{j}+\left(u^{p}\right)^{j} \frac{u_{x}^{j+1}-u_{x}^{j}}{\Delta t}\right] \\
& +\mathcal{O}\left(\Delta t^{2}\right) \\
& \simeq\left(u^{p}\right)^{j} u_{x}^{j+1}+p\left(u^{p-1}\right)^{j} u_{x}^{j} u^{j+1}-p\left(u^{p}\right)^{j} u_{x}^{j} .
\end{aligned}
$$

Now by plugging Eq. (8) into the Eq. (7) we obtain time discretized scheme as follows [16].

$$
\begin{array}{r}
u^{j+1} \\
+\Delta t \theta\left[u_{x}^{j+1}+\epsilon\left(\left(u^{p}\right)^{j} u_{x}^{j+1}+p\left(u^{p-1}\right)^{j} u_{x}^{j} u^{j+1}\right)\right] \\
-\mu\left(u_{x x}\right)^{j+1} \\
=u^{j}+\Delta t\left[\epsilon((p+1) \theta-1)\left(u^{p}\right)^{j} u_{x}^{j}-(1-\theta) u_{x}^{j}\right] \\
-\mu\left(u_{x x}\right)^{j}
\end{array}
$$

In numerical calculations we select $\theta=\frac{1}{2}$ which corresponds famous Crank-Nicolson approach.

\subsection{Space discretization with DBFs}

Let us assume the solution $u(x)$ can be approximated by the linear combination of DBFs as follows

$$
u^{j+1}(x)=\sum_{i=1}^{N} \lambda_{i}^{j+1} I_{M, \chi}\left(x, \xi_{i}\right) .
$$

Then first and second order derivatives can be found simply as

$$
\begin{aligned}
\frac{d}{d x} u^{j+1}(x) & =\sum_{i=1}^{N} \lambda_{i}^{j+1} \frac{d}{d x} I_{M, \chi}\left(x, \xi_{i}\right), \\
\frac{d^{2}}{d x^{2}} u^{j+1}(x) & =\sum_{i=1}^{N} \lambda_{i}^{j+1} \frac{d^{2}}{d x^{2}} I_{M, \chi}\left(x, \xi_{i}\right),
\end{aligned}
$$

Substituting Eqs. (10)-(12) into the Eq. (9) we obtain

$$
\begin{array}{r}
\sum_{i=1}^{N} \lambda_{i}^{j+1}\left(I_{M, \chi}\left(x, \xi_{i}\right)+\Delta t \theta\left[\frac{d}{d x} I_{M, \chi}\left(x, \xi_{i}\right)\right.\right. \\
\left.+\epsilon\left(\left(u^{p}\right)^{j} \frac{d}{d x} I_{M, \chi}\left(x, \xi_{i}\right)+p\left(u^{p-1}\right)^{j} u_{x}^{j} I_{M, \chi}\left(x, \xi_{i}\right)\right)\right] \\
\left.-\mu \frac{d^{2}}{d x^{2}} I_{M, \chi}\left(x, \xi_{i}\right)\right) \\
=\Delta t\left[\epsilon((p+1) \theta-1)\left(u^{p}\right)^{j} u_{x}^{j}-(1-\theta) u_{x}^{j}\right] \\
+u^{j}-\mu\left(u_{x x}\right)^{j}
\end{array}
$$

Discretizing Eq. (13) at collocation points $a=$ $x_{1}<x_{2}<\ldots<x_{N}=b$ and imposing boundary conditions (6) we can obtain a linear system of equations with size of $N \times N$ whose solution gives expansion coefficients $\lambda_{i}$. Then by using these coefficients in the (10) numerical solution can be found for each time step. The centers $\xi_{i}$ are different from collocation points but for convenience we take $\xi_{i}$ same as collocation points. For starting simulation, right hand side of the Eq. (13) must be calculated from initial condition.

We also should note that $I_{M, \chi}(x, \xi)$ vanishes near the boundaries $x= \pm 1$. Thus centers and collocation points should not be near the boundary in $[-1,1]$. To overcome this issue, as pointed out in [26], considered partial differential equations should be redefined in subdomain $[-0.5,0.5]$ by some scaling and transformation operations.

\section{Numerical experiments}

To indicate the performance of the proposed method we will use the error norms $L_{2}$ and $L_{\infty}$ defined by 


$$
\begin{aligned}
L_{2} & =\left\|u_{i}^{\text {exact }}-u_{i}^{\text {num }}\right\|_{2} \\
& \simeq\left(\Delta x \sum_{i=1}^{N}\left|u_{i}^{\text {exact }}-u_{i}^{\text {num }}\right|^{2}\right)^{1 / 2} \\
L_{\infty} & =\left\|u_{i}^{\text {exact }}-u_{i}^{\text {num }}\right\|_{\infty} \simeq \max _{i}\left|u_{i}^{\text {exact }}-u_{i}^{\text {num }}\right|
\end{aligned}
$$

and invariants [23]:

- Conservation of mass

$$
I_{1}=\int_{a}^{b} u d x=\Delta x \sum_{i=1}^{N} u_{i}
$$

- Conservation of momentum

$$
\begin{aligned}
I_{2} & =\int_{a}^{b}\left(u^{2}+\mu u_{x}^{2}\right) d x \\
& =\Delta x \sum_{i=1}^{N}\left[\left(u_{i}\right)^{2}+\mu\left(\left(u_{x}\right)_{i}\right)^{2}\right]
\end{aligned}
$$

- Conservation of energy

$I_{3}=\int_{a}^{b}\left(u^{4}-\mu u_{x}^{2}\right) d x=\Delta x \sum_{i=1}^{N}\left(u_{i}^{4}-\mu\left(\left(u_{x}\right)_{i}\right)^{2}\right)$.

Further, we calculate the convergence orders by the following formulae

$C_{1}=\frac{\log \left(\frac{L_{\infty, 2}(2 \Delta t, N)}{L_{\infty, 2}(\Delta t, N)}\right)}{\log 2}, \quad C_{2}=\frac{\log \left(\frac{L_{\infty, 2}(\Delta t, N)}{L_{\infty, 2}(\Delta t, 2 N)}\right)}{\log 2}$

We denote absolute differences of $I_{1}, I_{2}, I_{3}$ between initial time $t=0$ and final time $t=\mathrm{t}-$ final as $\left|\Delta I_{i}\right|=\left|I_{i}^{\mathrm{t}-\text { final }}-I_{i}^{\mathrm{t}-\text { initial }}\right|, \quad i=1,2,3$. In all numerical simulations we choose $\theta=0.5$ and we take $M=2 N+100, \chi=M / 40$ for single solitary wave problem, $M=2 N+300, \chi=M / 100$ for interaction of two-three solitary waves problem and $M=5 N+100, \chi=M / 100$ for Maxwellian problem. Numerical calculations have been done in Python environment 34, 35 with a desktop computer (Linux OS, NumPy version 1.15.1, Intel i7-8750H, 8GB RAM). Graphical outputs in this study were generated by Matplotlib package [36].

\subsection{Single solitary wave motion}

We investigate motion of single solitary given as

$$
u(x, 0)=\sqrt[p]{\frac{c(p+2)}{2 p} \sec h^{2}\left(\frac{p}{2} \sqrt{\frac{c}{\mu(c+1)}\left(x-x_{0}\right)}\right)} .
$$

To this end, we calculate the error norms $L_{2}, L_{\infty}$ and the invariants $I_{1}, I_{2}, I_{3}$ for constant values of $x_{0}=40, \mu=1,0 \leq x \leq 100$ and for various values of $\Delta t, c, p, N$. Firstly, to see convergence of the present method in space we fix $\Delta t=0.0001$ and we increase number of collocation points, obtained results are reported in Table 11. As it can be seen from the table by increasing number of collocation points the errors decrease. Later, we set $N=400$ and decrease time step size to see convergence in temporal variable. Obtained results are given in Table 2 where one can see that by halving the time step size the errors decrease and convergence orders are about two which is theoretical convergence order of Crank-Nicolson method.

In Table 3, for $N=100, c=0.1, \Delta t=0.05$ and $p=2,3$ the error norms are given at different final times with CPU times taken during simulation. Accuracy of the results can be seen from the table. Table 4 indicates variation in the invariants for $N=400, \Delta t=0.1, c=0.1$ and $p=2,3$ at different final times. From the table one can conclude that the proposed method can conserve invariants quite good.

In Tables 5 and 6 the invariants and errors are calculated and compared with ones of septic Bspline collocation method [24] for $\Delta t=0.01$, $\mu=1, p=4, c=0.3$ and $N=250$ (in case of the present method), $h=0.1$ (in case of the method of [24]). Absolute differences of $I_{1}, I_{2}, I_{3}$ between initial time $t=0$ and final time $t=10$ -are approximately $2 e-07,2 e-06,2 e-06$, respectively for the method of [24] while these differences are approximately $2 e-07,4 e-07,1.2 e-06$, respectively for the present method. In Table 7 , a comprehensive comparison between B-spline finite element 23, cubic B-spline collocation 6], Petrov-Galerkin [22], septic B-spline collocation 24 methods and the present method is given for $p=2,3,4$. For present method we take $N=250$ while for the other methods space step size $h$ is taken as 0.2 and 0.1. From the table it is clearly seen that for $p=3$ lowest errors are obtained by the present method and for $p=2,4$ lowest errors are obtained by the method of [24. Finally in Table 8 a comparison with compact finite difference [20] is given for $\Delta t=c=0.1$ where accuracy of the present method is obvious.

In Figs. 2 and 3, motion of single solitary waves are given for $p=3, c=1.2$ and $p=4, c=4 / 3$, respectively. It can be seen that at $t=0$ the solitary wave is located at $x_{0}=40$ and as time goes the single solitary wave moves rightward with constant speed and with almost invariable amplitude. 
Table 1. Error norms and convergence orders for $c=4 / 3, p=4$ and increasing values of $N$ at $t=0.1$.

\begin{tabular}{|c|c|c|c|c|}
\hline$N$ & $L_{2}$ & $L_{\infty}$ & $C_{2}$ for $L_{2}$ & $C_{2}$ for $L_{\infty}$ \\
\hline 40 & $7.154182 \mathrm{e}-01$ & $4.146875 \mathrm{e}-01$ & - & - \\
\hline 80 & $1.528925 \mathrm{e}-01$ & $1.253767 \mathrm{e}-01$ & 2.2263 & 1.7258 \\
\hline 160 & $1.141233 \mathrm{e}-03$ & $8.628014 \mathrm{e}-04$ & 7.0658 & 7.1830 \\
\hline 320 & $1.912428 \mathrm{e}-05$ & $2.386172 \mathrm{e}-05$ & 5.8990 & 5.1763 \\
\hline
\end{tabular}

Table 2. Error norms and convergence orders for $c=4 / 3, p=4$ and decreasing values of $\Delta t$ at $t=1$.

\begin{tabular}{|c|c|c|c|c|}
\hline$\Delta t$ & $L_{2}$ & $L_{\infty}$ & $C_{1}$ for $L_{2}$ & $C_{1}$ for $L_{\infty}$ \\
\hline $1 / 10$ & $5.540555 \mathrm{e}-02$ & $3.539277 \mathrm{e}-02$ & - & - \\
\hline $1 / 20$ & $1.409159 \mathrm{e}-02$ & $9.028174 \mathrm{e}-03$ & 1.9752 & 2.0136 \\
\hline $1 / 40$ & $3.494540 \mathrm{e}-03$ & $2.234878 \mathrm{e}-03$ & 2.0117 & 2.0142 \\
\hline $1 / 80$ & $8.792614 \mathrm{e}-04$ & $5.534720 \mathrm{e}-04$ & 1.9907 & 2.0136 \\
\hline
\end{tabular}

Table 3. Error norms and CPU times for $N=100, c=0.1, \Delta t=0.05, x_{0}=40$ on $0 \leq x \leq 100$ at different times.

\begin{tabular}{ccccccc}
\hline \multicolumn{2}{c}{$p=2$} & \multicolumn{2}{c}{$p=3$} & \\
\cline { 1 - 4 } Time & $L_{2}$ & $L_{\infty}$ & $L_{2}$ & $L_{\infty}$ & & CPU time \\
\hline$t=2$ & $1.396342 \mathrm{e}-05$ & $5.698786 \mathrm{e}-06$ & $3.618557 \mathrm{e}-05$ & $1.747289 \mathrm{e}-05$ & & 0.03 \\
$t=4$ & $2.732318 \mathrm{e}-05$ & $1.146938 \mathrm{e}-05$ & $7.126962 \mathrm{e}-05$ & $3.143534 \mathrm{e}-05$ & & 0.04 \\
$t=6$ & $4.059471 \mathrm{e}-05$ & $1.720380 \mathrm{e}-05$ & $1.060434 \mathrm{e}-04$ & $4.661107 \mathrm{e}-05$ & & 0.04 \\
$t=8$ & $5.360908 \mathrm{e}-05$ & $2.260812 \mathrm{e}-05$ & $1.401759 \mathrm{e}-04$ & $6.109345 \mathrm{e}-05$ & & 0.05 \\
$t=10$ & $6.632761 \mathrm{e}-05$ & $2.753908 \mathrm{e}-05$ & $1.736471 \mathrm{e}-04$ & $7.520650 \mathrm{e}-05$ & & 0.06 \\
\hline
\end{tabular}

Table 4. Invariants on $0 \leq x \leq 100$ for $N=400, \Delta t=0.1, c=0.1$ at different final times.

\begin{tabular}{ccccccccc}
\hline & \multicolumn{3}{c}{$p=2$} & & \multicolumn{3}{c}{$p=3$} & CPU time \\
\cline { 2 - 3 } \cline { 6 - 8 }$t$ & $I_{1}$ & $I_{2}$ & $I_{3}$ & & $I_{1}$ & $I_{2}$ & $I_{3}$ & \\
\hline 0 & 3.294918 & 0.683426 & 0.024121 & & 4.062584 & 1.133875 & 0.092900 & 0.00 \\
2 & 3.294919 & 0.683426 & 0.024121 & & 4.062584 & 1.133874 & 0.092899 & 0.33 \\
4 & 3.294920 & 0.683426 & 0.024121 & & 4.062585 & 1.133873 & 0.092899 & 0.38 \\
6 & 3.294919 & 0.683425 & 0.024121 & & 4.062585 & 1.133872 & 0.092898 & 0.42 \\
8 & 3.294919 & 0.683425 & 0.024121 & & 4.062584 & 1.133871 & 0.092896 & 0.48 \\
10 & 3.294918 & 0.683425 & 0.024121 & & 4.062583 & 1.133871 & 0.092895 & 0.53 \\
\hline
\end{tabular}

Table 5. Invariants and their comparison on $0 \leq x \leq 100$ for $N=250, \Delta t=0.01, \mu=1, p=4$, $c=0.3$

\begin{tabular}{|c|c|c|c|c|c|c|}
\hline & $\begin{array}{l}24] \text { (second) } \\
\end{array}$ & Present & [24] (second) & Present & $\begin{array}{l}24] \text { (second) } \\
\end{array}$ & Present \\
\hline$t$ & $I_{1}$ & $I_{1}$ & $I_{2}$ & $I_{2}$ & $I_{3}$ & $I_{3}$ \\
\hline 0 & 3.7592865 & 3.7592300 & 1.7300239 & 1.7300029 & 0.2894189 & 0.2894090 \\
\hline 2 & 3.7592865 & 3.7592300 & 1.7300244 & 1.7300028 & 0.2894183 & 0.2894091 \\
\hline 4 & 3.7592865 & 3.7592299 & 1.7300250 & 1.7300027 & 0.2894178 & 0.2894097 \\
\hline 6 & 3.7592864 & 3.7592299 & 1.7300254 & 1.7300026 & 0.2894174 & 0.2894100 \\
\hline 8 & 3.7592864 & 3.7592299 & 1.7300256 & 1.7300025 & 0.2894171 & 0.2894101 \\
\hline 10 & 3.7592863 & 3.7592298 & 1.7300259 & 1.7300024 & 0.2894169 & 0.2894102 \\
\hline
\end{tabular}

\subsection{The interaction of two solitary waves}

In this subsection, we examine interaction of two solitary waves, namely we consider the Eq. (2) $u(x, 0)=\sum_{i=1}^{2} \sqrt[p]{\frac{c_{i}(p+2)}{2 p} \sec h^{2}\left(\frac{p}{2} \sqrt{\frac{c_{i}}{\mu\left(c_{i}+1\right)}\left(x-x_{i}\right)}\right)}$ with following initial condition 
Table 6. Invariants and their comparison on $0 \leq x \leq 100$ for $N=250, \Delta t=0.01, \mu=1, p=4$, $c=0.3$

\begin{tabular}{cccccccc}
\hline & {$[24]$ (first) } & {$[24]$ (second) } & Present & & [24] (first) & {$[24]$ (second) } & Present \\
\cline { 2 - 6 }$t$ & $L_{2} \times 10^{4}$ & $L_{2} \times 10^{4}$ & $L_{2} \times 10^{4}$ & & $L_{\infty} \times 10^{4}$ & $L_{\infty} \times 10^{4}$ & $L_{\infty} \times 10^{4}$ \\
\hline 2 & 0.25417530 & 0.19937853 & 0.2803098 & & 0.13193138 & 0.09833776 & 0.1510377 \\
4 & 0.50867400 & 0.39600506 & 0.5629237 & & 0.25511505 & 0.19527926 & 0.2957829 \\
6 & 0.76378746 & 0.59159317 & 0.8494472 & & 0.37848569 & 0.29108460 & 0.4345260 \\
8 & 1.01967310 & 0.78622772 & 1.1406822 & & 0.50227119 & 0.38611041 & 0.5756090 \\
10 & 1.27628477 & 0.98004530 & 1.4373113 & & 0.62645346 & 0.48083798 & 0.7138410 \\
\hline
\end{tabular}

Table 7. Comparison of the results on $0 \leq x \leq 100$ for $\mu=1$ at $t=10$.

\begin{tabular}{|c|c|c|c|c|}
\hline & & $p=2, c=1$ & $p=3, c=0.3$ & $p=4, c=0.3$ \\
\hline & & $\Delta t=0.025, h=0.2$ & $\Delta t=0.01, h=0.1$ & $\Delta t=0.01, h=0.1$ \\
\hline \multirow[t]{10}{*}{$I_{1}$} & Present method, $N=250$ & 4.44288292 & 3.67755181 & 3.75922990 \\
\hline & Collocation+PA-CN (cubic) 23$]$ & 4.44000000 & - & - \\
\hline & Collocation-CN (cubic) 23 & 4.44200000 & - & - \\
\hline & Collocation (cubic) [6] & 4.44288000 & - & - \\
\hline & Petrov-Galerkin (quintic) 22] & 4.44288000 & 3.67755000 & 3.75923000 \\
\hline & Collocation (septic) 24 & 4.44286610 & 3.67760690 & 3.75928630 \\
\hline & Present method, $N=250$ & 3.29978116 & 1.56574072 & 1.73000240 \\
\hline & Collocation+PA-CN (cubic) 23. & 3.29600000 & - & - \\
\hline & Collocation-CN (cubic) 23] & 3.29900000 & - & - \\
\hline & Collocation (cubic) 6] & 3.29983000 & - & - \\
\hline \multirow[t]{5}{*}{$I_{2}$} & Petrov-Galerkin (quintic) 22] & 3.29981000 & 1.56574000 & 1.72999000 \\
\hline & Collocation (septic) 24 & 3.29971510 & 1.56576200 & 1.73002590 \\
\hline & Present method, $N=250$ & 1.41416306 & 0.22683878 & 0.28941022 \\
\hline & Collocation+PA-CN (cubic) 23 & 1.41100000 & - & - \\
\hline & Collocation-CN (cubic) 23] & 1.41300000 & - & - \\
\hline \multirow[t]{6}{*}{$I_{3}$} & Collocation (cubic) 6] & 1.41420000 & - & - \\
\hline & Petrov-Galerkin (quintic) 22] & 1.41416000 & 0.22683700 & 0.28940600 \\
\hline & Collocation (septic) 24 & 1.41431220 & 0.22684460 & 0.28941690 \\
\hline & Present method, $N=250$ & 3.91431278 & 0.06900426 & 0.14368290 \\
\hline & Collocation+PA-CN (cubic) 23 & 20.30000000 & - & - \\
\hline & Collocation-CN (cubic) 23] & 16.39000000 & - & - \\
\hline \multirow[t]{6}{*}{$L_{2} \times 10^{3}$} & Collocation (cubic) 6] & 9.30196000 & - & - \\
\hline & Petrov-Galerkin (quintic) 22 & 3.00533000 & 0.07197600 & 0.12253900 \\
\hline & Collocation (septic) 24 & 2.57148152 & 0.07851367 & 0.09800453 \\
\hline & Present method, $N=250$ & 2.00191759 & 0.03304418 & 0.07169059 \\
\hline & Collocation+PA-CN (cubic) 23$]$ & 11.20000000 & - & - \\
\hline & Collocation-CN (cubic) 23] & 9.24000000 & - & - \\
\hline \multirow[t]{3}{*}{$L_{\infty} \times 10^{3}$} & Collocation (cubic) 6 & 5.43718000 & - & - \\
\hline & Petrov-Galerkin (quintic) [22] & 1.68749000 & 0.03772280 & 0.06620700 \\
\hline & Collocation (septic) 24 & 1.34021078 & 0.03650124 & 0.04808379 \\
\hline
\end{tabular}

which describes propagation of two waves with different amplitudes, one placed at $x_{1}$ and the other placed at $x_{2}$.

First numerical simulation have been done with the following values $p=2, c_{1}=4, c_{2}=1, x_{1}=$ $25, x_{2}=55, \Delta t=0.025, \mu=1$ on the interval $0 \leq x \leq 250$. The results obtained are reported in Table 9 and are compared with PetrovGalerkin 22 and septic B-spline collocation 24 methods.
From the table we can see that the invariants obtained by the present method are compatible with the ones of 22, 24]. In Fig. 4, interaction of the solitary waves are depicted.

Second simulation have been done with $p=3$, $c_{1}=48 / 5, c_{2}=6 / 5, x_{1}=20, x_{2}=50, \Delta t=0.01$, $0 \leq x \leq 120$ and $\mu=1$. The obtained results are reported and compared with the results of 22 and 24] in Table 10. Variations in the invariants $I_{1}, I_{2}, I_{3}$ are approximately $2.0 e-06,0.111$, 
Table 8. Comparison of the results on $0 \leq x \leq 100, \mu=1, x_{0}=40$ and $\Delta t=c=0.1$.

\begin{tabular}{|c|c|c|c|c|c|c|c|c|c|}
\hline & & \multicolumn{4}{|c|}{$p=1$} & \multicolumn{4}{|c|}{$p=2$} \\
\hline & & $I_{1}$ & $I_{2}$ & $L_{2}$ & $L_{\infty}$ & $I_{1}$ & $I_{2}$ & $L_{2}$ & $L_{\infty}$ \\
\hline \multirow[t]{2}{*}{$t=2$} & $20, h=0.1$ & 1.989963 & 0.196378 & 0.013774 & 0.005403 & 3.29492 & 0.649425 & 0.039859 & 0.018973 \\
\hline & Present, $N=250$ & 1.989964 & 0.202616 & 0.000011 & 0.000004 & 3.294919 & 0.683426 & 0.000055 & 0.000023 \\
\hline \multirow[t]{2}{*}{$t=4$} & $20, h=0.1$ & 1.989964 & 0.197220 & 0.012347 & 0.004610 & 3.29492 & 0.653939 & 0.036136 & 0.015780 \\
\hline & Present, $N=250$ & 1.989965 & 0.202616 & 0.000022 & 0.000008 & 3.294920 & 0.683426 & 0.000109 & 0.000047 \\
\hline \multirow[t]{2}{*}{$t=6$} & $20], h=0.1$ & 1.989964 & 0.198076 & 0.010985 & 0.003841 & 3.29492 & 0.658616 & 0.032839 & 0.013296 \\
\hline & Present, $N=250$ & 1.989965 & 0.202616 & 0.000032 & 0.000012 & 3.294919 & 0.683425 & 0.000162 & 0.000070 \\
\hline \multirow[t]{2}{*}{$t=8$} & $20, h=0.1$ & 1.989963 & 0.198947 & 0.009737 & 0.003158 & 3.29492 & 0.663465 & 0.030230 & 0.011791 \\
\hline & Present, $N=250$ & 1.989964 & 0.202616 & 0.000043 & 0.000016 & 3.294919 & 0.683425 & 0.000214 & 0.000093 \\
\hline \multirow[t]{2}{*}{$t=10$} & $20, h=0.1$ & 1.989962 & 0.199832 & 0.008677 & 0.002656 & 3.29492 & 0.668494 & 0.028541 & 0.011065 \\
\hline & Present, $N=250$ & 1.989963 & 0.202616 & 0.000053 & 0.000020 & 3.294918 & 0.683425 & 0.000265 & 0.000113 \\
\hline
\end{tabular}

$p=3$

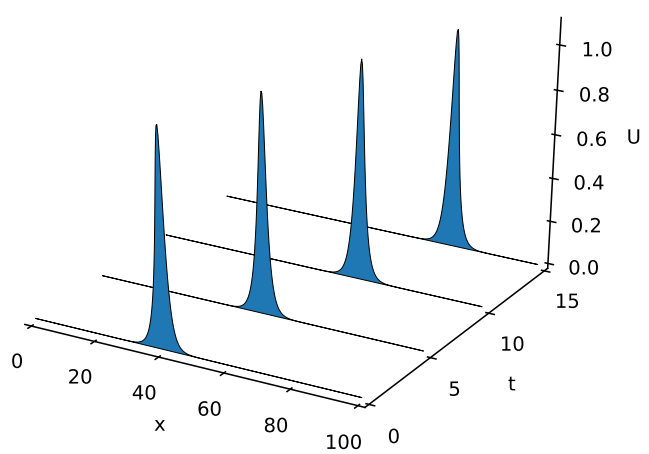

Figure 2. Motion of single solitary wave at $t=0,5,10,15$ for $N=400$, $\Delta t=0.05$ and $p=3$.

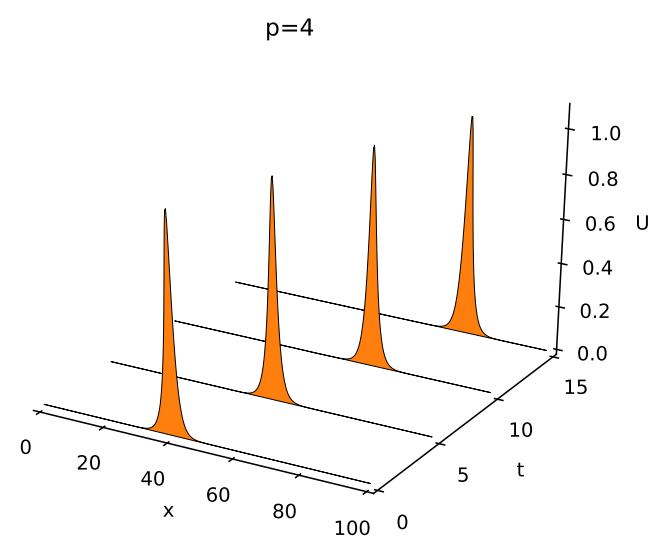

Figure 3. Motion of single solitary wave at $t=0,5,10,15$ for $N=400$, $\Delta t=0.05$ and $p=4$.

Table 9. Comparison of the results for $p=2, c_{1}=4, c_{2}=1, x_{1}=25, x_{2}=55, \Delta t=0.025$, $\mu=1$ on $0 \leq x \leq 250$ at different final times.

\begin{tabular}{cccccccc}
\hline & & $t=0$ & $t=4$ & $t=8$ & $t=12$ & $t=16$ & $t=20$ \\
\hline$I_{1}$ & Present method, $N=400$ & 11.4676982 & 11.4677197 & 11.4676926 & 11.4676587 & 11.4676037 & 11.4674483 \\
& 24] Collocation (second), $h=0.2$ & 11.4676542 & 11.4676484 & 11.4668849 & 11.4676777 & 11.4676555 & 11.4676452 \\
& 22] Petrov-Galerkin (quintic), $h=0.2$ & 11.4677000 & 11.4677000 & 11.4677000 & 11.4677000 & 11.4677000 & 11.4677000 \\
\hline \multirow{3}{*}{$I_{2} \quad$ 24] Collocation (second), $h=0.2$} & 14.6292089 & 14.6277880 & 14.1400014 & 14.6803731 & 14.6442435 & 14.6309639 \\
& Present method, $N=400$ & 14.6290652 & 14.6194206 & 14.6068263 & 14.6029824 & 14.5933673 & 14.5831979 \\
& 22] Petrov-Galerkin (quintic), $h=0.2$ & 14.6286000 & 14.6292000 & 14.6229000 & 14.6299000 & 14.6295000 & 14.6299000 \\
\hline & Present method, $N=400$ & 22.8816460 & 22.8411085 & 22.7875495 & 22.7753681 & 22.7381963 & 22.6975609 \\
& 24] Collocation (second), $h=0.2$ & 22.8803575 & 22.8817784 & 23.3695650 & 22.8291933 & 22.8653229 & 22.8786025 \\
& 22] Petrov-Galerkin (quintic), $h=0.2$ & 22.8788000 & 22.8811000 & 22.8798000 & 22.8803000 & 22.8805000 & 22.8806000 \\
\hline
\end{tabular}

0.45 respectively for the present method. Fig. 10 shows the interaction of the solitary waves.

\subsection{The interaction of three solitary waves}

The Eq. (2) with initial condition

$$
u(x, 0)=\sum_{i=1}^{3} \sqrt[p]{\frac{c_{i}(p+2)}{2 p} \sec h^{2}\left(\frac{p}{2} \sqrt{\frac{c_{i}}{\mu\left(c_{i}+1\right)}\left(x-x_{i}\right)}\right)}
$$




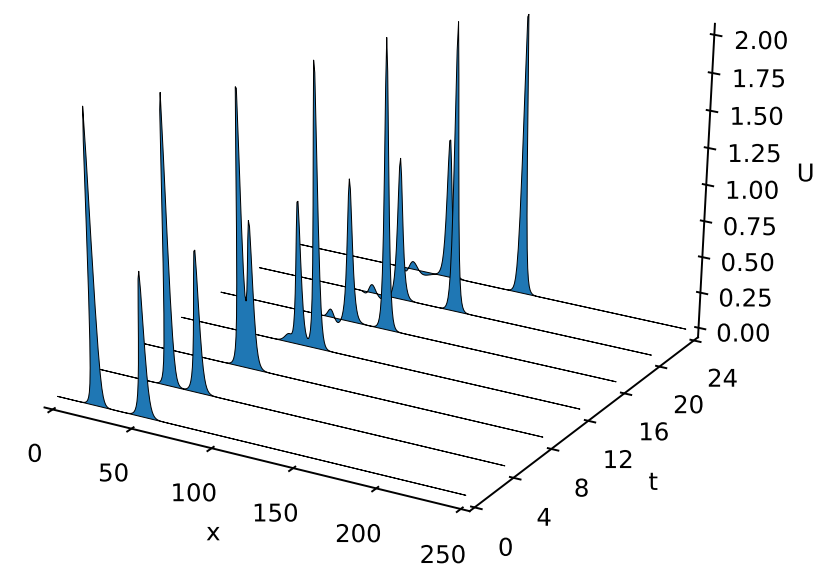

Figure 4. Interaction of two solitary waves for $N=400, p=2, c_{1}=4, c_{2}=1, x_{1}=25$, $x_{2}=55, \Delta t=0.025$, and $\mu=1$ at $t=0,4,8,12,16,20$.

Table 10. Comparison of the results for $p=3, c_{1}=48 / 5, c_{2}=6 / 5, x_{1}=20, x_{2}=50$, $\Delta t=0.01, \mu=1$ on $0 \leq x \leq 120$ at different final times.

\begin{tabular}{cccccccc}
\hline & & $t=0$ & $t=2$ & $t=3$ & $t=4$ & $t=5$ & $t=6$ \\
\hline$I_{1}$ & Present method, $N=400$ & 9.6907416 & 9.6907408 & 9.6907405 & 9.6907403 & 9.6907398 & 9.6907396 \\
& 24] Collocation (second), $h=0.1$ & 9.6907772 & 9.6881175 & 9.6850972 & 9.6860154 & 9.6847993 & 9.6834620 \\
& 22] Petrov-Galerkin (quintic), $h=0.1$ & 9.6907500 & 9.6907400 & 9.6907400 & 9.6907400 & 9.6907400 & 9.6907400 \\
\hline \multirow{3}{*}{$I_{2}$} & Present method, $N=400$ & 12.9443811 & 12.9034856 & 12.8814687 & 12.8721151 & 12.8526253 & 12.8331028 \\
& 24] Collocation (second), $h=0.1$ & 12.9443914 & 12.9390629 & 12.3046064 & 12.9703128 & 13.0538036 & 13.0027533 \\
& 22] Petrov-Galerkin (quintic), $h=0.1$ & 12.9444000 & 12.9452000 & 12.9379000 & 12.9453000 & 12.9457000 & 12.9454000 \\
\hline \multirow{3}{*}{$I_{3}$} & Present method, $N=400$ & 17.0187240 & 16.8733431 & 17.5959108 & 16.7459006 & 16.5917866 & 16.5602450 \\
& 24] Collocation (second), $h=0.1$ & 17.0186758 & 17.0240043 & 17.6584608 & 16.9927544 & 16.9092637 & 16.9603139 \\
& 22] Petrov-Galerkin (quintic), $h=0.1$ & 17.0184000 & 16.9835000 & 17.0591000 & 16.9261000 & 16.8781000 & 16.9113000 \\
\hline
\end{tabular}

is considered in this subsection. The above initial condition describes movement of three solitary waves with different amplitudes in same direction. For numerical simulation, we choose $0 \leq x \leq 100$, $\mu=1, c_{1}=0.6, c_{2}=0.3, c_{3}=0.15, x_{1}=15$, $x_{2}=35, x_{3}=60$ and different values of $\Delta t$ and $p$. In Table [1], we calculate the invariants for $N=400, p=2, \Delta t=0.1$ and compare the results with compact finite difference method [20. In the same table we give absolute difference of the invariants approximately, between initial time $t=0$ and final time $t=10$ where it can be seen that the present method conserves invariants better than the method of [20]. In Tables 12, 13 the invariants and their changes are given for $N=400$, $\Delta t=0.05$ and $p=3,4$ respectively. From these tables we can conclude that the present method can conserve invariants successfully. Finally the interaction of three solitary waves are shown in Figs. 6 and 7 .

\subsection{Maxwellian inital condition}

Finally, in this subsection we consider the Eq.(2) with

$$
u(x, 0)=e^{-\left(x^{2}\right),} \quad-20 \leq x \leq 60
$$

Maxwellian initial condition. In this case, it is known that solution depends on $\mu$ [15,21]. Let us assume $\mu_{c}$ be some critical value. If $\mu \gg \mu_{c}$ then the solution shows rapidly oscillating behavior without breaking up into solitons. When $\mu<\mu_{c}$ the solution forms solitons based on the value of $\mu$. Lastly if $\mu=\mu_{c}$ a leading soliton with oscillating tail occurs. We perform numerical simulations for various values of $\mu=0.1,0.050 .025,0.01$. In first simulation we consider the case $p=3$. We 


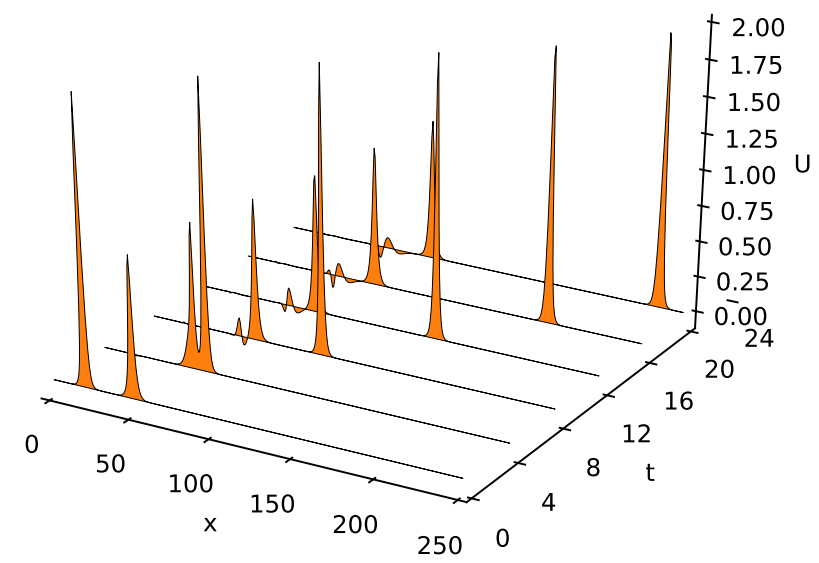

Figure 5. Interaction of two solitary waves for $N=400, p=3, c_{1}=48 / 5, c_{2}=6 / 5, x_{1}=20$, $x_{2}=50, \Delta t=0.025$, and $\mu=1$ at $t=0,4,8,12,16,20$.

Table 11. Invariants and their changes for $p=2$ and $\Delta t=0.1$.

\begin{tabular}{|c|c|c|c|c|c|c|}
\hline & \multicolumn{3}{|c|}{ [20], $h=0.1$} & \multicolumn{3}{|c|}{ Present, $N=400$} \\
\hline$t$ & $I_{1}$ & $I_{2}$ & $I_{3}$ & $I_{1}$ & $I_{2}$ & $I_{3}$ \\
\hline 0 & 10.9245 & 4.4191 & 0.740798 & 10.9245437 & 4.4191243 & 0.7407977 \\
\hline 2 & 10.9245 & 3.8743 & 0.505953 & 10.9246354 & 4.4190394 & 0.7407469 \\
\hline 4 & 10.9246 & 4.0302 & 0.573556 & 10.9246419 & 4.4189527 & 0.7406946 \\
\hline 6 & 10.9245 & 4.2342 & 0.669611 & 10.9246165 & 4.4188661 & 0.7406418 \\
\hline 8 & 10.9245 & 4.5023 & 0.812142 & 10.9245872 & 4.4187793 & 0.7405883 \\
\hline 10 & 10.9244 & 4.8697 & 1.039870 & 10.9245403 & 4.4186918 & 0.7405338 \\
\hline$\left|\Delta I_{i}\right| \rightarrow$ & $1.0 \mathrm{e}-04$ & 0.45060 & 0.29907 & $3.4 \mathrm{e}-06$ & $4.325 \mathrm{e}-04$ & $2.639 \mathrm{e}-04$ \\
\hline
\end{tabular}

Table 12. Invariants and their changes for $p=3, N=400$ and $\Delta t=0.05$.

\begin{tabular}{|c|c|c|c|c|}
\hline$t$ & $I_{1}$ & $I_{2}$ & $I_{3}$ & CPU time \\
\hline$\overline{0}$ & 11.1945795 & 4.8882472 & 0.7971747 & 0.00 \\
\hline 2 & 11.1946512 & 4.8881944 & 0.7972071 & 0.25 \\
\hline 4 & 11.1946551 & 4.8881413 & 0.7972680 & 0.34 \\
\hline 6 & 11.1946323 & 4.8880883 & 0.7973291 & 0.43 \\
\hline 8 & 11.1946040 & 4.8880352 & 0.7974067 & 0.51 \\
\hline 10 & 11.1945561 & 4.8879819 & 0.7975176 & 0.60 \\
\hline$\left|\Delta I_{i}\right| \rightarrow$ & $9.5100 \mathrm{e}-05$ & $2.6530 \mathrm{e}-04$ & $3.4290 \mathrm{e}-04$ & \\
\hline
\end{tabular}

Table 13. Invariants and their changes for $p=4, N=400$ and $\Delta t=0.05$.

\begin{tabular}{|c|c|c|c|c|}
\hline$t$ & $I_{1}$ & $I_{2}$ & $I_{3}$ & CPU time \\
\hline 0 & 11.4706872 & 5.3297106 & 0.9191609 & 0.00 \\
\hline 2 & 11.4707529 & 5.3295569 & 0.9192072 & 0.25 \\
\hline 4 & 11.4707560 & 5.3294031 & 0.9193171 & 0.35 \\
\hline 6 & 11.4707341 & 5.3292498 & 0.9194077 & 0.42 \\
\hline 8 & 11.4707057 & 5.3290969 & 0.9195143 & 0.51 \\
\hline 10 & 11.4706562 & 5.3289442 & 0.9196717 & 0.61 \\
\hline$\left|\Delta I_{i}\right| \rightarrow$ & $3.1000 \mathrm{e}-05$ & $7.6640 \mathrm{e}-04$ & $5.1080 \mathrm{e}-04$ & \\
\hline
\end{tabular}

take $N=400, \Delta t=0.005$ for the present method. We give changes in the invariants and their comparison with results of septic B-spline collocation 


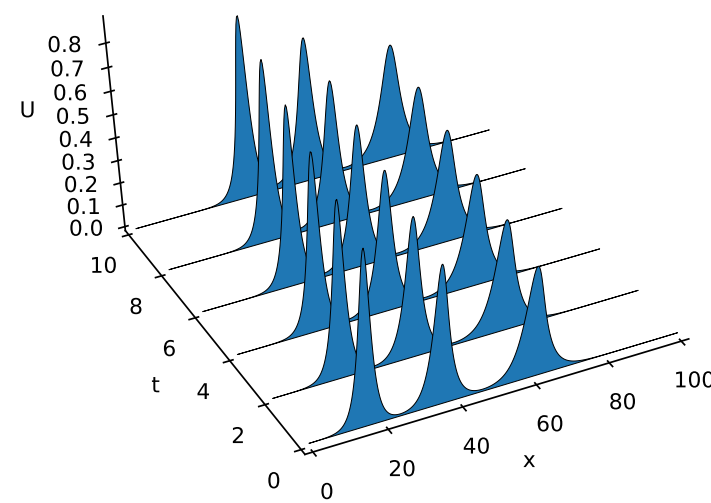

Figure 6. Interaction of three solitary waves for $p=3, N=400, \mu=1, c_{1}=$ $0.6, c_{2}=0.3, c_{3}=0.15, x_{1}=15, x_{2}=$ 35 and $x_{3}=60$ at $t=0,2,4,6,8,10$.

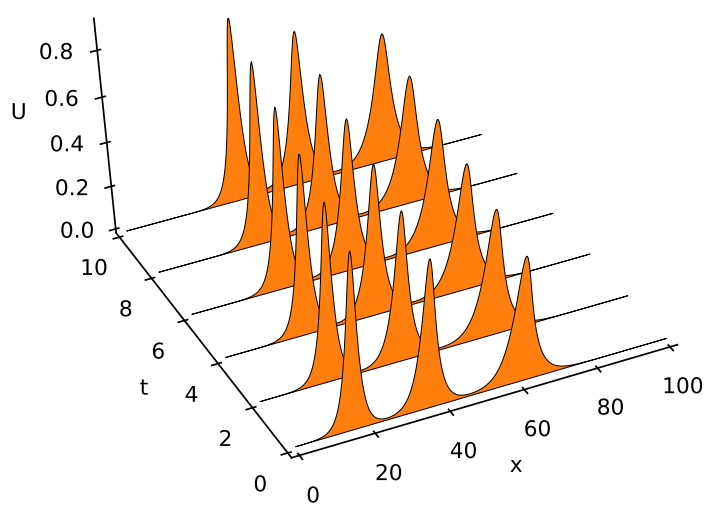

Figure 7. Interaction of three solitary waves for $p=4, N=400, \mu=1, c_{1}=$ $0.6, c_{2}=0.3, c_{3}=0.15, x_{1}=15, x_{2}=$ 35 and $x_{3}=60$ at $t=0,2,4,6,8,10$.

Table 14. Invariants and their comparison for $p=3, N=400, \Delta t=0.005$ and different values of $\mu$.

\begin{tabular}{|c|c|c|c|c|c|c|c|c|c|}
\hline \multicolumn{10}{|c|}{$p=3$} \\
\hline & & Present & 24 & & Present & 24 & & Present & 24 \\
\hline & $t$ & $I_{1}$ & $I_{1}$ & & $I_{2}$ & $I_{2}$ & & $I_{3}$ & $I_{3}$ \\
\hline \multirow[t]{4}{*}{$\mu=0.1$} & 0 & 1.772454 & 1.772453 & & 1.378646 & 1.378645 & & 0.760896 & 0.760895 \\
\hline & 2 & 1.772766 & 1.772452 & & 1.379399 & 1.548191 & & 0.607357 & 0.591349 \\
\hline & 4 & 1.772561 & 1.772451 & & 1.378142 & 1.546329 & & 0.604010 & 0.593211 \\
\hline & 6 & 1.772610 & 1.772449 & \multirow[b]{2}{*}{$\left|\Delta I_{2}\right| \rightarrow$} & 1.378273 & 1.545540 & \multirow[b]{2}{*}{$\left|\Delta I_{3}\right| \rightarrow$} & 0.603203 & 0.594000 \\
\hline$\left|\Delta I_{1}\right| \rightarrow$ & & 0.000156 & $4.0 \mathrm{e}-06$ & & 0.000373 & 0.166895 & & 0.157693 & 0.166895 \\
\hline \multirow[t]{4}{*}{$\mu=0.05$} & 0 & 1.772454 & 1.772453 & & 1.315980 & 1.315979 & \multirow[b]{5}{*}{$\left|\Delta I_{3}\right| \rightarrow$} & 0.823561 & 0.823561 \\
\hline & 2 & 1.772215 & 1.772376 & & 1.312421 & 1.514843 & & 0.639867 & 0.624697 \\
\hline & 4 & 1.772022 & 1.772272 & & 1.311619 & 1.514131 & & 0.639441 & 0.625409 \\
\hline & 6 & 1.773135 & 1.772168 & & 1.317028 & 1.513035 & & 0.648906 & 0.626505 \\
\hline$\left|\Delta I_{1}\right| \rightarrow$ & & 0.000681 & 0.000285 & $\left|\Delta I_{2}\right| \rightarrow$ & 0.001048 & 0.197056 & & 0.174655 & 0.197056 \\
\hline \multirow[t]{4}{*}{$\mu=0.025$} & 0 & 1.772454 & 1.772453 & \multirow[b]{5}{*}{$\left|\Delta I_{2}\right| \rightarrow$} & 1.284647 & 1.284646 & \multirow[b]{5}{*}{$\left|\Delta I_{3}\right| \rightarrow$} & 0.854894 & 0.854894 \\
\hline & 2 & 1.782801 & 1.768943 & & 1.332664 & 1.502469 & & 0.815844 & 0.637071 \\
\hline & 4 & 1.774529 & 1.764956 & & 1.302657 & 1.501801 & & 0.754045 & 0.637740 \\
\hline & 6 & 1.754215 & 1.761477 & & 1.222551 & 1.498994 & & 0.589541 & 0.640546 \\
\hline$\left|\Delta I_{1}\right| \rightarrow$ & & 0.018239 & 0.010976 & & 0.062096 & 0.214348 & & 0.265353 & 0.214348 \\
\hline \multirow[t]{4}{*}{$\mu=0.01$} & 0 & 1.772454 & 1.772453 & \multirow[b]{5}{*}{$\left|\Delta I_{2}\right| \rightarrow$} & 1.265847 & 1.265847 & \multirow[b]{5}{*}{$\left|\Delta I_{3}\right| \rightarrow$} & 0.873694 & 0.873693 \\
\hline & 2 & 1.733125 & 1.720433 & & 1.172092 & 1.456451 & & 0.616309 & 0.683090 \\
\hline & 4 & 1.711463 & 1.706008 & & 1.120066 & 1.450265 & & 0.541076 & 0.689276 \\
\hline & 6 & 1.731412 & 1.700567 & & 1.196719 & 1.451593 & & 0.733945 & 0.687947 \\
\hline$\left|\Delta I_{1}\right| \rightarrow$ & & 0.041042 & 0.071886 & & 0.069128 & 0.185746 & & 0.139749 & 0.185746 \\
\hline
\end{tabular}

method in Table 14. Further, for $p=4$ the results obtained are reported in Table 15,

Graphics of numerical solutions for various values of parameter $\mu$ are given in Figs. 8 and 9, Breaking of solitons can be observed from the Figs. 8 and 9 .

\section{Conclusion}

In this paper, delta-shaped functions combined with the finite difference and a linearization approach are used for numerically solving generalized regularized long wave equation.

The present method has been tested on four classic problems and its accuracy has been assessed 
Table 15. Invariants and their comparison for $p=4, N=400, \Delta t=0.005$ and different values of $\mu$

\begin{tabular}{|c|c|c|c|c|c|c|c|c|c|}
\hline & & & & & $p=4$ & & & & \\
\hline & & Present & 24 & & Present & 24 & & Present & 24 \\
\hline & $t$ & $I_{1}$ & $I_{1}$ & & $I_{2}$ & $I_{2}$ & & $I_{3}$ & $I_{3}$ \\
\hline \multirow[t]{4}{*}{$\mu=0.1$} & 0 & 1.772454 & 1.772453 & & 1.378646 & 1.378645 & & 0.760896 & 0.760895 \\
\hline & 2 & 1.772594 & 1.772110 & & 1.376700 & 1.591837 & & 0.474657 & 0.547703 \\
\hline & 4 & 1.772315 & 1.771702 & & 1.375158 & 1.588948 & & 0.467131 & 0.550592 \\
\hline & 6 & 1.774090 & 1.771297 & & 1.381579 & 1.587779 & \multirow[b]{2}{*}{$\left|\Delta I_{3}\right| \rightarrow$} & 0.469617 & 0.551761 \\
\hline$\left|\Delta I_{1}\right| \rightarrow$ & & 0.001636 & 0.001156 & $\left|\Delta I_{2}\right| \rightarrow$ & 0.002933 & 0.209134 & & 0.291279 & 0.209134 \\
\hline \multirow[t]{4}{*}{$\mu=0.05$} & 0 & 1.772454 & 1.772453 & & 1.315980 & 1.315979 & \multirow[b]{5}{*}{$\left|\Delta I_{3}\right| \rightarrow$} & 0.823561 & 0.823561 \\
\hline & 2 & 1.765624 & 1.753662 & & 1.293771 & 1.535874 & & 0.512686 & 0.603666 \\
\hline & 4 & 1.772599 & 1.741625 & & 1.321959 & 1.528679 & & 0.551366 & 0.610862 \\
\hline & 6 & 1.755506 & 1.733910 & & 1.265309 & 1.523490 & & 0.481081 & 0.616050 \\
\hline$\left|\Delta I_{1}\right| \rightarrow$ & & 0.016948 & 0.038543 & $\left|\Delta I_{2}\right| \rightarrow$ & 0.05067 & 0.207511 & & 0.342480 & 0.207511 \\
\hline \multirow[t]{4}{*}{$\mu=0.025$} & 0 & 1.772454 & 1.772453 & & 1.284647 & 1.284646 & \multirow[b]{5}{*}{$\left|\Delta I_{3}\right| \rightarrow$} & 0.854894 & 0.854894 \\
\hline & 2 & 1.789069 & 1.693029 & & 1.355683 & 1.482414 & & 0.739884 & 0.657126 \\
\hline & 4 & 1.711672 & 1.682425 & & 1.133213 & 1.476250 & & 0.412816 & 0.663290 \\
\hline & 6 & 1.714808 & 1.674869 & & 1.141655 & 1.468703 & & 0.409811 & 0.670837 \\
\hline$\left|\Delta I_{1}\right| \rightarrow$ & & 0.057646 & 0.0975840 & $\left|\Delta I_{2}\right| \rightarrow$ & 0.142992 & 0.184057 & & 0.445083 & 0.184057 \\
\hline \multirow[t]{4}{*}{$\mu=0.01$} & 0 & 1.772454 & 1.772453 & & 1.265847 & 1.265847 & \multirow[b]{5}{*}{$\left|\Delta I_{3}\right| \rightarrow$} & 0.873694 & 0.873693 \\
\hline & 2 & 1.825320 & 1.651315 & & 1.464030 & 1.437490 & & 1.304239 & 0.702051 \\
\hline & 4 & 1.750123 & 1.644999 & & 1.261045 & 1.439995 & & 0.843022 & 0.699545 \\
\hline & 6 & 1.761501 & 1.633634 & & 1.294508 & 1.431710 & & 0.929302 & 0.707830 \\
\hline$\left|\Delta I_{1}\right| \rightarrow$ & & 0.010953 & 0.138819 & $\left|\Delta I_{2}\right| \rightarrow$ & 0.028661 & 0.165863 & & 0.055608 & 0.165863 \\
\hline
\end{tabular}
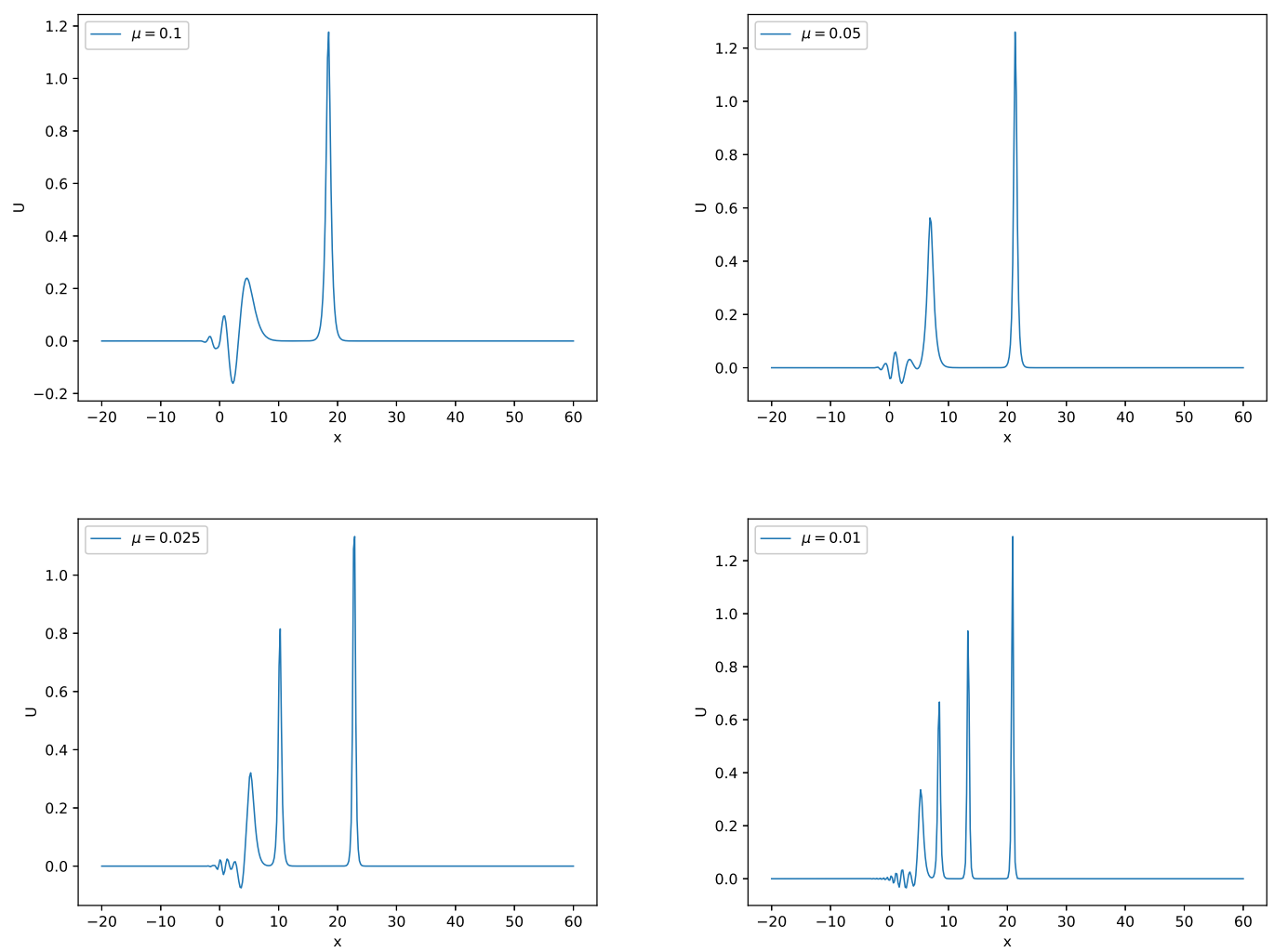

Figure 8. Numerical solution for $p=3, N=400, \Delta t=0.005$ and different values of $\mu$ at $t=6$.

by comparing calculated error norms $L_{2}, L_{\infty}$ and invariants $I_{1}, I_{2}, I_{3}$ with exact values and with finite element, finite difference and collocation methods. It is seen that from calculations 

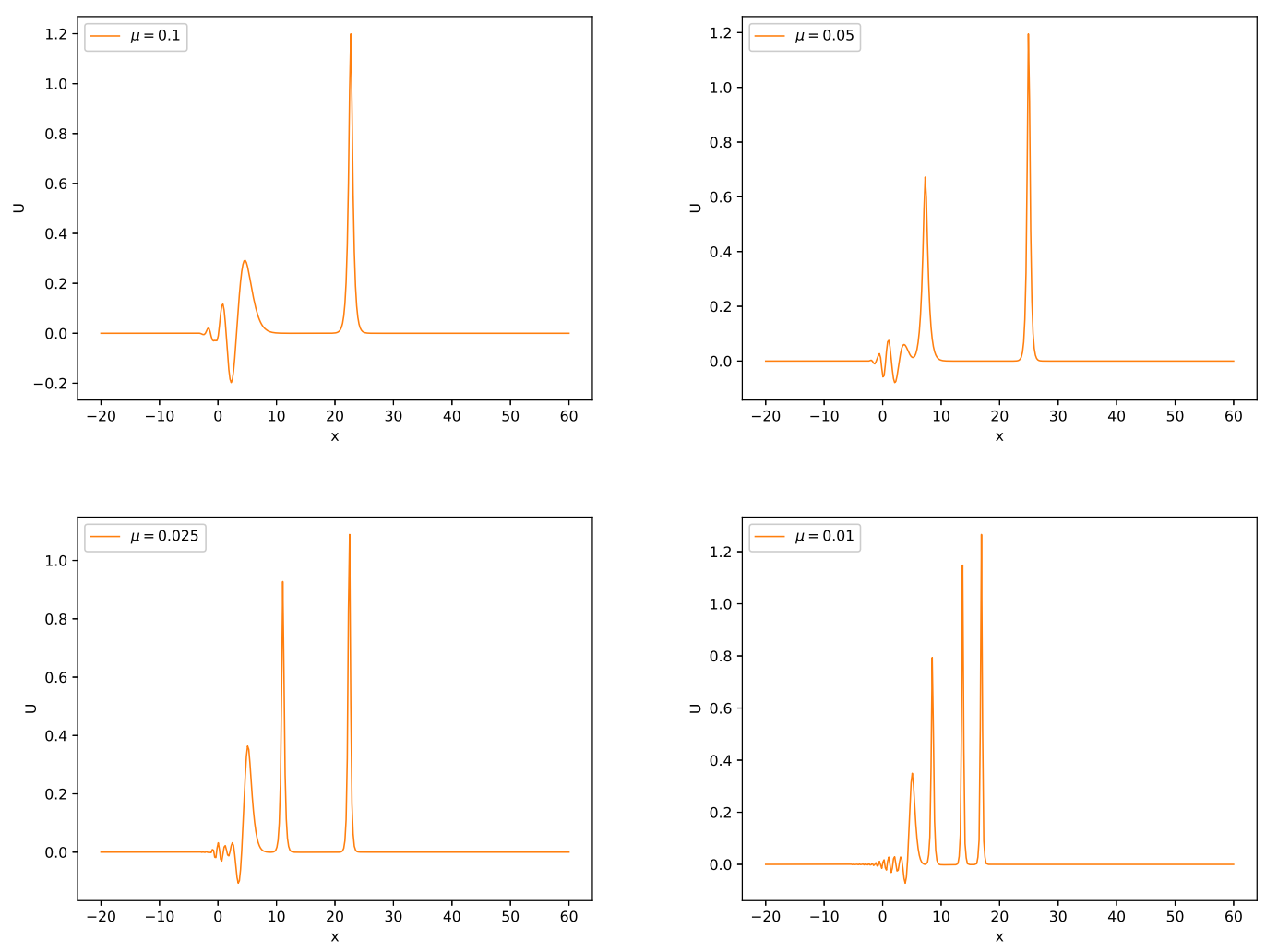

Figure 9. Numerical solution for $p=4, N=400, \Delta t=0.005$ and different values of $\mu$ at $t=6$.

the invariants are almost constant during numerical simulations and error norms are satisfactorily good even in less collocation points. The performance of the present method indicates that the present method is competitive with existing methods such as finite element method, finite difference and collocation methods. Furthermore, the performance of the present method encourages us to use the method for other nonlinear partial differential equations that have applications in various engineering and scientific fields.

\section{Acknowledgments}

The author receives no fund for this research.

\section{References}

[1] Garcia-Lopez, C.M., \& Ramos, J.I.(2012). Effects of convection on a modified GRLW equation, Applied Mathematics and Computation, 219(8), 4118-4132.

[2] Peregrine, D. H.(1966). Calculations of the development of an undular bore, Journal of Fluid Mechanics, 25, 321-330.

[3] Peregrine, D. H. (1967). Long waves on a beach, Journal of Fluid Mechanics, 27(4), 815-827.

[4] Benjamin, T. B., Bona, J. L., \& Mahony, J. J. (1972). Model equations for long waves in non-linear dispersive systems, Philosophical Transactions of the Royal Society of London A, 272, 47-78.

[5] Abdulloev, Kh. O., Bogalubsky, H., \& Markhankov, V.G.(1976). One more example of inelastic soliton interaction, Physics Letters A, 56, 427-428.

[6] Khalifa, A.K., Raslan, K.R., \& Alzubaidi, H.M.(2008). A collocation method with cubic b-splines for solving the MRLW equation, Journal of Computational and Applied Mathematics, 212(2), 406-418

[7] Shokri, A., \& Dehghan, M.(2010). A meshless method using the radial basis functions for numerical solution of the regularized long wave equation, Numerical Methods for Partial Differential Equations, 26(4), 807-825

[8] Oruç, Ö., Bulut, F., \& Esen, A.(2016). Numerical Solutions of Regularized Long Wave Equation By Haar Wavelet Method, Mediterranean Journal of Mathematics, 13(5), 32353253.

[9] Dehghan, M., \& Salehi, R.(2011). The solitary wave solution of the two-dimensional regularized long-wave equation in fluids and plasmas, Computer Physics Communications, 182, 2540-2549. 
[10] Dehghan, M., Abbaszadeh, M., \& Mohebbi, A.(2015). The use of interpolating elementfree Galerkin technique for solving 2D generalized Benjamin-Bona-Mahony-Burgers and regularized long wave equations on nonrectangular domains with error estimate, Journal of Computational and Applied Mathematics, 286, 211-231.

[11] Dag, I., Irk, D., \& Sari, M. (2013). The extended cubic b-spline algorithm for a modified regularized long wave equation, Chinese Physics B, 22(4), doi: 10.1088/ 16741056/22/4/040207.

[12] Karakoç, S.B.G., Yagmurlu, N.M., \& Ucar, Y. (2013) Numerical approximation to a solution of the modified regularized long wave equation using quintic b-splines, Bound. Value Probl. 2013 27, doi: 10.1186/16872770- 2013- 27.

[13] Esen, A., \& Kutluay, S.(2006). Application of a lumped Galerkin method to the regularized long wave equation, Applied Mathematics and Computation, 174, 833-845.

[14] Dogan, A.(2002). Numerical solution of RLW equation using linear finite elements within Galerkins method, Applied Mathematical Modelling, 26, 771-783.

[15] Kaya, D.(2004). A numerical simulation of solitary-wave solutions of the generalized regularized long wave equation, Applied Mathematics and Computation, 149, 833-841.

[16] Mokhtari, R., \& Mohammadi, M.(2010) Numerical solution of GRLW equation using Sinc-collocation method, Computer Physics Communications, 181(7), 1266-1274.

[17] Wang, J.-F., Bai, F.-N., \& Cheng, Y.M.(2011). A meshless method for the nonlinear generalized regularized long wave equation, Chinese Physics B, 20(3), 030206.

[18] Guo, P., Zhang, L., \& Liew, K.(2014). Numerical analysis of generalized regularized long wave equation using the element-free kpRitz method, Applied Mathematics and Computation, 240, 91-101.

[19] Kang, X., Cheng, K., \& Guo, C.(2015). A second-order Fourier pseudospectral method for the generalized regularized long wave equation, Advances in Difference Equations, 2015 (1), 339, doi: 10.1186/s13662- 015- 06763.

[20] Hammad, D.A., \& El-Azab, M.S.(2015). A $2 \mathrm{~N}$ order compact finite difference method for solving the generalized regularized long wave (GRLW) equation, Applied Mathematics and Computation 253, 248-261
[21] Zhang, L.(2005). A finite difference scheme for generalized regularized long-wave equation, Applied Mathematics and Computation, 168, 962-972.

[22] Roshan, T.(2012). A Petrov-Galerkin method for solving the generalized regularized long wave (GRLW) equation, Computers \& Mathematics with Applications, 63, 943-956

[23] Gardner, L.R.T., Gardner, G.A., Ayoub, F.A., \& Amein, N.K.(1997). Approximations of solitary waves of the MRLW equation by b-spline finite element, Arabian Journal for Science and Engineering 22, 183-193.

[24] Karakoc, S.B.G., \& Zeybek, H.(2016). Solitary-wave solutions of the GRLW equation using septic B-spline collocation method, Applied Mathematics and Computation, 289, 159-171

[25] Li, Q., \& Mei, L.(2018). Local momentumpreserving algorithms for the GRLW equation, Applied Mathematics and Computation 330, 77-92

[26] Reutskiy, S.Y.(2005). A boundary method of Trefftz type for PDEs with scattered data. Engineering Analysis with Boundary Elements, 29, 713-24.

[27] Tian, H.Y., Reustkiy, S., \& Chen, C.S.(2007). New basis functions and their applications to PDEs. In: ICCES, vol. 3(4), 169-75.

[28] Tian, H.Y., Reustkiy, S., \& Chen, C.S.(2008). A basis function for approximation and the solutions of partial differential equations, Numerical Methods for Partial Differential Equations, 24(3), 1018-36.

[29] Hon, Y.C., \& Yang, Z.(2009). Meshless collocation method by Delta-shape basis functions for default barrier model. Engineering Analysis with Boundary Elements, 33, 951-958

[30] Reutskiy, S.Y.(2011). A meshless method for one-dimensional Stefan problems, Applied Mathematics and Computation, 217, 9689-9701

[31] Mokhtari, R., Isvand, D., Chegini, N.G., \& Salaripanah, A.(2013). Numerical solution of the Schrödinger equations by using Deltashaped basis functions, Nonlinear Dynamics, 74, 77-93

[32] Tian, H., \& Grunewalda, A.(2017). A DeltaShaped Basis Method for Ill-Posed Nonhomogeneous Elliptic Boundary Value Problems, Neural, Parallel, and Scientific Computations, 25, 1-18

[33] Oruç, Ö., Two meshless methods based on pseudo spectral delta-shaped basis functions 
and barycentric rational interpolation for numerical solution of modified Burgers equation, International Journal of Computer Mathematics, DOI: 10.1080/00207160.2020.1755432

[34] Oliphant, T.E.(2007). Python for Scientific Computing, Computing In Science \& Engineering, 9 (3), 10-20.

[35] Walt, S. van der, Colbert S. C., \& Varoquaux, G. (2011) The NumPy Array: A Structure for Efficient Numerical Computation, Computing In Science \& Engineering, 13 (2), 22-30.
[36] Hunter, J. D.(2007). Matplotlib: A 2D graphics environment, Computing In Science \& Engineering, 9(3), 90-95.

Ömer Oruç obtained his M.Sc. degree in fuzzy differential equations from department of Mathematics, TOBB Economics and Technology University in 2011, and his Ph. D. in Haar wavelet based numerical methods from department of Mathematics, Inonu University in 2016. His current research areas include fuzzy theory, differential equations, numerical methods and scientific computing. He has over 20 papers published in SCI and SCI-Expanded indexed journals.

(iD http://orcid.org/0000-0002-6655-3543

An International Journal of Optimization and Control: Theories \& Applications (http://ijocta.balikesir.edu.tr)

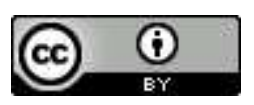

This work is licensed under a Creative Commons Attribution 4.0 International License. The authors retain ownership of the copyright for their article, but they allow anyone to download, reuse, reprint, modify, distribute, and/or copy articles in IJOCTA, so long as the original authors and source are credited. To see the complete license contents, please visit http://creativecommons.org/licenses/by/4.0/. 